\title{
Lymph node involvement and metastatic lymph node ratio influence the survival of malignant pleural mesothelioma: A French multicenter retrospective study
}

\author{
ILIR HYSI $^{1}$, FRANÇOISE LE PIMPEC-BARTHES ${ }^{2}$, MARCO ALIFANO $^{3}$, NICOLAS VENISSAC $^{4}$, \\ JÉRÔME MOUROUX ${ }^{4}$, JEAN-FRANÇOIS REGNARD $^{3}$, MARC RIQUET $^{2}$ and HENRI PORTE ${ }^{1}$ \\ ${ }^{1}$ Department of Thoracic Surgery, Albert Calmette Hospital, Lille; ${ }^{2}$ Department of Thoracic Surgery, \\ Georges Pompidou European Hospital, AP-HP, Paris; ${ }^{3}$ Department of Thoracic Surgery, \\ Hôtel-Dieu Hospital, AP-HP, Paris; ${ }^{4}$ Department of Thoracic Surgery, \\ Louis Pasteur Hospital, Nice, France
}

Received August 7, 2013; Accepted September 16, 2013

DOI: $10.3892 / o r .2013 .2800$

\begin{abstract}
Malignant pleural mesothelioma (MPM) is a rare tumor with disastrous evolution. The prognostic value of nodal involvement is still debated. We analyzed the impact of nodal involvement on overall survival (OS) in patients treated by multimodal therapy including extra pleural pneumonectomy (EPP). We evaluated the role, as a prognostic factor, of the metastatic lymph node ratio (LNR), corresponding to the number of involved nodes out of the total number of removed nodes. In this retrospective multicentric study, we reviewed the data of 99 MPM patients. Information regarding lymph node involvement was assessed from the final pathology reports. N1-N3 patients were pooled as $\mathrm{N}+$ group. The OS, calculated by the Kaplan-Meier method, was compared using the log-rank test. A multivariate Cox proportional hazards model was used to identify independent prognostic factors. For the whole cohort, median OS was 18.3 months and 5-year survival was $17.5 \%$. N+ status reduced significantly the median survival (22.4 months for N0 patients vs 12.7 months for $\mathrm{N}+$ patients, $\mathrm{P}=0.002)$. A lower metastatic LNR $(\leq 13 \%)$ was associated with a significantly improved median survival (19.9 vs. 11.7 months, $\mathrm{P}=0.01$ ). OS was not related to the number of involved or total removed lymph nodes. In multivariate analysis, only adjuvant radiotherapy $(\mathrm{P}=0.001)$ was identified as an independent positive prognostic factor. Metastatic LNR is a more reliable prognostic factor than the number of involved lymph nodes or the total number of removed nodes. However, it could not be identified as an independent prognostic factor.
\end{abstract}

Correspondence to: Dr Ilir Hysi, Department of Thoracic Surgery, Albert Calmette Hospital, F59037 Lille, France

E-mail: ilirhysi@gmail.com

Key words: malignant pleural mesothelioma, lymph node, ratio, multicenter

\section{Introduction}

Malignant pleural mesothelioma (MPM) is an asbestos related neoplasm, associated with poor prognosis. Its incidence is projected to increase in Europe with a peak around 2020 and with approximately 250,000 expected deaths in the next 40 years (1). Although the MARS trial (2), despite its questionable methodology, recently showed disappointing results of surgery, some multimodal treatment studies, evaluating surgery [extrapleural pneumonectomy (EPP) or pleurectomy/decortication (P/D)] plus chemoradiotherapy showed satisfactory results in terms of survival, with a median ranging from 9.4 to 27.5 months (3).

Prognostic assessment is especially based on stages of disease. Different staging systems have been used over the past years for MPM (4), the most current being the TNM staging system proposed in 1995 by the International Mesothelioma Interest Group (IMIG) (5). In this classification the nodal staging is the same as for lung cancer, despite the differences in lymph spread pattern between MPM [which involves almost uniformly the parietal pleura (6)] and lung carcinoma (7). The prognostic value of nodal involvement in MPM is debated. Many authors (8-11) showed the N+ status to be associated with poor outcome, whereas others $(12,13)$ observed no significant difference between N+ and N0 patients. Moreover, the $\mathrm{N}+$ group is heterogeneous, with respect to topography and extent. Adjustments of pathological staging, taking differently into account the $\mathrm{N}$ factor, have been recently proposed (14). In particular, the N1 status remains unsettled as some authors suggest classifying it as lower stage (8) and others suggest upgrading it to higher stage (15). Multiple N2 disease and involvement of $\geq 4$ nodes are also considered as negative prognostic factors $(8,9)$.

In several cancers, including lung and breast carcinoma (16), the number of involved lymph nodes has been shown to be associated with survival. Given the known anatomic variation in lymph node numbers between patients, according to BMI and preoperative treatments, the metastatic lymph node ratio (LNR), which is more reliable as not affected 
by these variations, has been proposed as a prognostic factor in rectal, esophageal and lung cancer (16-18). The aim of this study was to evaluate the nodal involvement impact on survival and to assess the role of metastatic LNR as a prognostic factor in MPM. This study was conducted studying patients from four French centers with similar strategy in management of patients with MPM.

\section{Materials and methods}

Patient population. This retrospective study was conducted by analyzing data from patients treated between December, 1997 and September, 2010 in four French Thoracic Surgery Departments of University Hospitals [Cochin-Hôtel-Dieu Hospital (Paris), Georges Pompidou European Hospital (Paris), University Hospital of Nice (Nice) and University Hospital of Lille (Lille)]. During this period, 99 patients underwent extrapleural pneumonectomy (EPP) in a curative intent for MPM formally diagnosed by biopsy. Individual data were assessed from clinical and pathological charts. Information regarding the pattern of nodal involvement was analyzed from the final histological findings. The pTNM IMIG stage classification (5) was used. This study was approved by the Institutional Review Board of the French Society of Thoracic and Cardiovascular Surgery (CERC-SFCTCV-2012-9-25-11-19-52-HyIl) and patients consent requirement was waived.

Preoperative work-up. Patients were considered to be operable after a thorough work-up including physical examination, physiological evaluation and imaging. All of them underwent contrast-enhanced CT scans of the thorax and upper abdomen. At the beginning of 2002 a PET-CT scan was progressively performed in a standard manner. When axial mediastinal nodes involvement was uncertain (enlarged or hypermetabolic), a mediastinoscopy was carried out to ascertain that these nodes were free of invasion. Cerebral CT scan was performed according to the surgeons and preference of the centers in a non-systematic manner. Neither laparoscopy nor contralateral thoracoscopy was performed for staging purposes.

Surgical approach. EPP was performed through a posterolateral thoracotomy as part of a multimodal treatment including chemotherapy, radiotherapy and in one center hyperthermic intrathoracic chemotherapy either as neoadjuvant or adjuvant treatment. The lung, pleura, pericardium and diaphragm were excised en bloc and reconstruction of the pericardium and diaphragm was performed as described by Wolf et al (19). A systematic nodal dissection was performed according to the technique described by Graham et al (20), supplemented by a systematic internal mammary and pericardial fat node dissection. Detailed information regarding lymph nodes was collected from the final pathological findings. The number, location and possible capsular rupture of lymph nodes, as described by the pathologist of each center, were carefully assessed. Lymph nodes were classified in different stations and chains according to the Naruke map (21) and Riquet definitions (22), respectively. Hilar nodes were classified as N1, internal mammary nodes $\mathrm{N} 2$ and supraclavicular nodes $\mathrm{N} 3$. Because of a small number, N1-N3 node-positive patients were pooled as $\mathrm{N}+$ patients in further analyses. Metastatic LNR, expressed as a percentage, was calculated as the ratio between total positive and removed nodes.

Hyperthermic intrathoracic chemotherapy. This technique was used as part of a tetramodal treatment in only one center (Nice). After a double regimen of cisplatin/pemetrexed chemotherapy, standard surgery was performed. Upon completion of EPP resection and diaphragmatic reconstruction, before thorax closure, the patient was given HIT chemotherapy with cisplatin $100 \mathrm{mg} / \mathrm{m}^{2}$ and gemcitabine $1,250 \mathrm{mg} / \mathrm{m}^{2}$ for $1 \mathrm{~h}$ at $42^{\circ} \mathrm{C}$, which was associated to a renal protective intravenous perfusion of amifostin. A peritoneal tube was not associated to this procedure as described by Mujoomdar et al (23). Radiotherapy up to 54 Gy was administered as adjuvant therapy.

Follow-up. All patients surviving operation were followed-up. Events were defined as death before 31 May, 2012. Diagnosis of recurrence was generally based on histological evidence. Operative mortality included all patients who succumbed to the disease within 90 days of surgery. Survival was calculated from the date of surgery until the death or the end of the follow-up termination time (right censoring) including postoperative mortality. Survival data were available for all patients.

Statistical analysis. Data are shown as absolute number and percentage, means or median (SD/range). Correlation was expressed by the Spearman's rank coefficient. Continuous data were compared by Mann-Whitney U test and ordinal data by the $\chi^{2}$ test or Fisher's exact test as appropriate. The cut-off for the total number of lymph nodes dissected, number of positive lymph nodes, number of metastatic nodal stations and metastatic LNR was calculated by a data-oriented method [25th, 50th (median), 75th percentile]. Concerning the cut-off for metastatic LNR it was defined at the 75 th percentile (value $\leq 13 \%$ ) as at the median the LNR value was 0 . Survival was calculated by the Kaplan-Meier method including postoperative deaths and comparisons in univariate analysis were carried out by log-rank test. Factors identified at univariate analysis as significantly associated with survival were entered in a multistep Cox multivariate model to assess independent factors. Statistics Epidémiology Medecine (SEM) software (version 3.5; Centre Jean Perrin, Clermont-Ferrand, France) was used for statistical analysis and GraphPad Prism 5 software (Graphpad Software Inc., La Jolla, CA, USA) was used to generate graphic presentations of the results.

\section{Results}

Patient characteristics. Main patient characteristics are shown in Table I. The mean age at presentation was $58.7( \pm 7.5)$ years. Smoking habits in the cohort showed a mean tobacco consumption of $16.7( \pm 19.5)$ pack years. Most patients (84.8\%) had no relevant medical history. Detailed data on preoperative work-up were available for 62 patients. PET-CT scans were performed in $47(75.8 \%)$ of them and brain CT scans in $27(43.5 \%)$ patients. In addition, 15 (24.1\%) patients had undergone mediastinoscopy to rule out a preoperative 
Table I. Patient clinical and treatment characteristics.

\begin{tabular}{lc}
\hline Characteristic & $\mathrm{n}(\%)$ \\
\hline Male & $83(83.4)$ \\
Center & \\
1 & $30(30.3)$ \\
2 & $29(29.3)$ \\
4 & $28(28.3)$ \\
3 & $12(12.1)$
\end{tabular}

Occupation

NA data

$45(45.4)$

Building worker

$18(33.3)$

Steelworker

Others

Asbestos exposure

$13(24.1)$

$23(42.6)$

$75(81.5)$

Smokers

58 (58.6)

ASA

NA data

1

2

3

PS

0

1

$41(41.4)$

18 (31)

$32(55.1)$

8 (13.9)

$72(72.7)$

27 (27.3)

Clinical symptoms at diagnosis

NA data

Pleural effusion

Parietal pain

Chronic bronchitis

Spontaneous pneumothorax

Neoadjuvant therapy

None

$20(22.9)$

Chemotherapy

$67(77.1)$

Operation

Right sided

R0 surgery

$56(56.5)$

93 (94)

Adjuvant therapy

None

$20(20.8)$

HIT chemotherapy (perioperative)

$12(12.5)$

1 (1.1)

Chemotherapy

Radiotherapy

63 (65.6)

Histology

Epitheloid

Non-epitheloid

$86(86.8)$

13 (13.2)

Stage

I

II

24 (24.2)

III

$61(61.6)$

IV

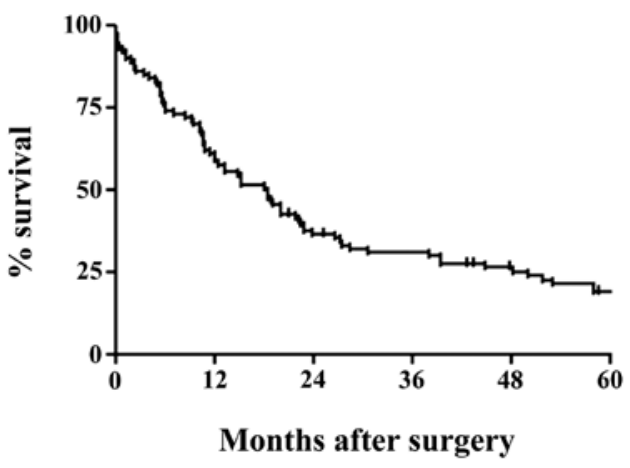

Figure 1. Overall survival.

$\mathrm{N} 2$ status. The neoadjuvant treatment consisted mainly of 2 or 3 rounds of cisplatin/pemetrexed. Pathologic stages were pT1 in 10 patients (10.1\%), pT2 in 34 (34.3\%), pT3 in 51 (51.5\%) and pT4 in $4(4.1 \%)$ of them.

Lymph node dissection and LNR. The mean number of total lymph nodes in the mediastinal dissection was 11.9 $( \pm 6.4)$. There was no correlation between the total number of dissected nodes and different clinicopathological factors assessed (weight, neoadjuvant treatment, surgery side), however, it tended to be higher in taller patients $(\mathrm{P}=0.08)$. The nodal status of patients was as follows: $65(65.6 \%)$ N0, 3 $(3.1 \%) \mathrm{N} 1,30(30.3 \%) \mathrm{N} 2$ and $1(1 \%) \mathrm{N} 3$. The N3 patient was a unique case with a solitary skip subclavicular positive lymph node. The details of the invasion of lymph node stations are presented in Table II. Among the 15 patients who had mediastinoscopy with negative findings, two had pN2 at the final result, but with positive nodes in the extra axial mediastinum, internal mammary and parietal nodes.

Metastatic LNR was $\leq 13 \%$ in $75(75.7 \%)$ patients. In the whole population (including both $\mathrm{N} 0$ and $\mathrm{N}+$ patients) mean LNR was $11.1 \%( \pm 22.03 \%)$. Among patients with $\mathrm{N}+$ disease, mean LNR was $32.3 \%( \pm 26.9 \%)$.

Survival analysis. The median follow-up was 84.7 months (range, 21-176) and it was complete for all patients. Median overall survival (OS) was 18.3 months and the 5-year survival was $17.5 \%$ (Fig. 1). Table III resumes univariate and multivariate analyses for OS. In the univariate analysis there was a significant difference in OS with respect to PS categories ( $\mathrm{P}=0.02$; Fig. 2A). Although R0 resections were performed more frequently in patients who underwent neoadjuvant treatment $(\mathrm{P}=0.009)$, neither neoadjuvant treatment $(\mathrm{P}=0.09)$ nor HIT chemotherapy $(\mathrm{P}=0.38)$ significantly determined OS. As for the surgical procedure, patients with $\mathrm{R} 0$ resection had a better survival $(\mathrm{P}=0.02$; Fig. $2 \mathrm{~B})$. The pTNM staging was also an important prognostic factor. Survival was higher in early disease $(\mathrm{P}=0.04$; Fig. $2 \mathrm{C})$, in $\mathrm{N} 0$ patients $(\mathrm{P}=0.002$; Fig. 2D) and in patients with a metastatic $\mathrm{LNR} \leq 13 \%(\mathrm{P}=0.01$; Fig. 2E). OS was significantly related to epitheloid histology $(\mathrm{P}=0.009)$ and adjuvant radiotherapy $(\mathrm{P}=0.01$; Fig. $2 \mathrm{~F})$. For the entire cohort, at the cut-off value of $\leq 13 \%$ there was a significantly different survival with respect to LNR $(\mathrm{P}=0.01)$. This survival difference disappeared in the subgroup of $\mathrm{N}+$

patients $(\mathrm{P}=0.88)$.
1, Cochin-Hotel-Dieu Hospital; 2, Georges Pompidou European Hospital; 3, Lille Hospital; 4, Nice Hospital; NA, not available; ASA, American Society of Anesthesiologists; PS, performance status. 
Table II. Detailed nodal information in N2/N3 patients.

\begin{tabular}{lc}
$\mathrm{n}(\%$ of $\mathrm{N} 2 / \mathrm{N} 3$ patients $)$ & $\mathrm{n}(\%$ of all the patients $)$ \\
\hline Right & Teft
\end{tabular}

\section{Nodal stations}

$$
\begin{aligned}
& 2 \\
& 3 \\
& 4 \\
& 5 / 6 \\
& 7 \\
& 8 \\
& 9
\end{aligned}
$$$$
3
$$$$
7
$$

Parietal nodes

Internal mammary nodes

One-level nodal disease

Two-level nodal disease $\geq$ Three-level nodal disease

Capsular rupture
$1(3.2)$

1 (3.2)

7 (22.5)

-

9 (29)

2 (6.4)

1 (3.2)

3 (9.6)

6 (19.3)

6 (19.3)

5 (16.1)

8 (25.8)

4 (12.9)

-
-
$3(9.6)$
$5(16.1)$
$8(25.8)$
$2(6.4)$
$3(9.6)$
$2(6.4)$
$6(19.3)$
$4(12.9)$
$4(12.9)$
$7(22.5)$
$5(16.1)$

1 (1)

1 (1)

$10(10.1)$

5 (5)

17 (17.1)

4 (4)

4 (4)

5 (5)

12 (12.1)

$10(10.1)$

$9(9.1)$

15 (15.1)

9 (9.1)

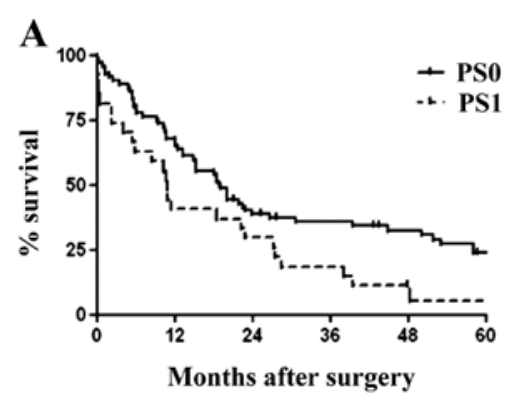

C

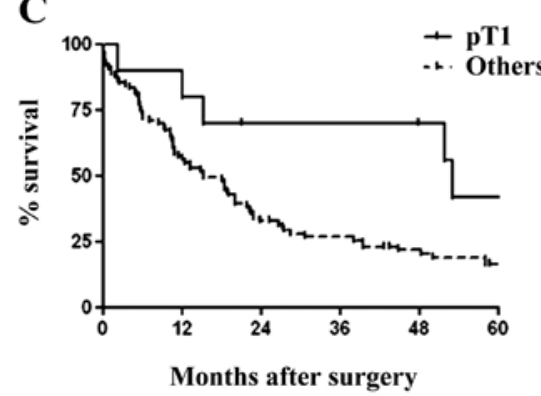

$\mathbf{E}$

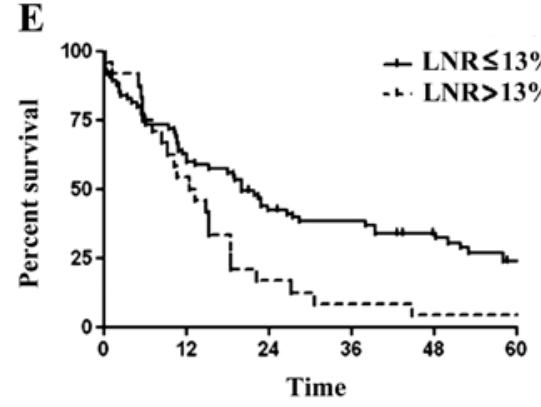

B

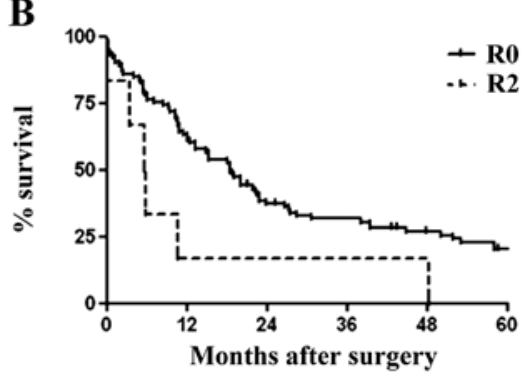

D

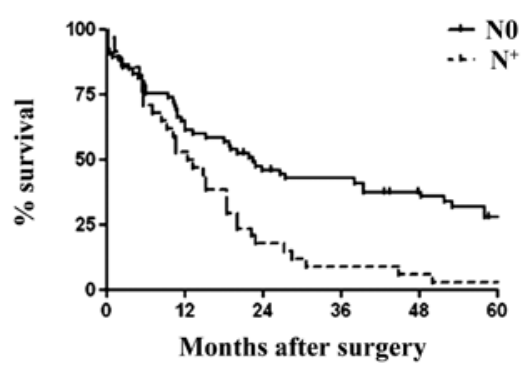

F

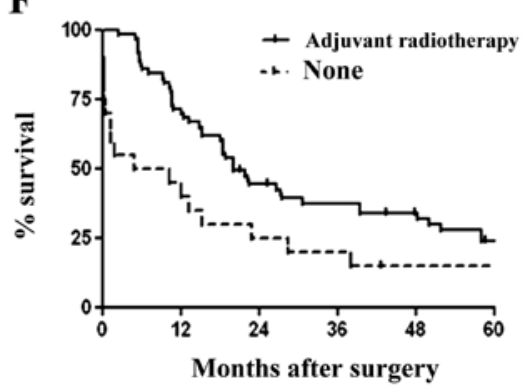

Figure 2. Overall survival by (A) performance status, (B) surgical resection, (C) pT, (D) pN, (E) metastatic LNR and (F) adjuvant treatment.

In the multivariate analysis only adjuvant radiotherapy was a robust independent prognostic factor. Exploratory analysis allowed the identification of a subset of patients with better outcome: for patients with NO status who sustained adjuvant 
Table III. Univariate and multivariate analysis of prognostic factors for overall survival.

\begin{tabular}{|c|c|c|}
\hline \multirow[b]{2}{*}{ Factors } & \multicolumn{2}{|c|}{ Univariate analysis } \\
\hline & $\begin{array}{l}\text { Median } \\
\text { (months) }\end{array}$ & $\begin{array}{c}\text { Log-rank } \\
\text { P-value }\end{array}$ \\
\hline \multicolumn{3}{|l|}{ Gender } \\
\hline Male & 18.3 & 0.93 \\
\hline Female & 16.9 & \\
\hline \multicolumn{3}{|l|}{ PS } \\
\hline PS0 & 18.9 & 0.02 \\
\hline PS1 & 10.7 & \\
\hline \multicolumn{3}{|l|}{ Neoadjuvant treatment } \\
\hline Administrated & 18.7 & 0.06 \\
\hline Non administrated & 11.9 & \\
\hline \multicolumn{3}{|c|}{ Perioperative HIT chemotherapy } \\
\hline Administrated & 17.9 & 0.34 \\
\hline Non administrated & 22 & \\
\hline \multicolumn{3}{|l|}{ Surgical resection } \\
\hline R0 & 18.4 & 0.02 \\
\hline $\mathrm{R} 2$ & 5.6 & \\
\hline \multicolumn{3}{|l|}{ pT } \\
\hline pT1 & 52.9 & 0.04 \\
\hline Others pT & 15.2 & \\
\hline \multicolumn{3}{|l|}{$\mathrm{pN}$} \\
\hline pN0 & 22.4 & 0.002 \\
\hline $\mathrm{pN}+$ & 12.7 & \\
\hline \multicolumn{3}{|c|}{ Total no. of lymph nodes dissected } \\
\hline$\leq 10$ & 14.7 & 0.24 \\
\hline$>10$ & 18 & \\
\hline \multicolumn{3}{|c|}{ No. of positive lymph nodes } \\
\hline$\leq 3$ & 17.2 & 0.38 \\
\hline$>3$ & 18.4 & \\
\hline \multicolumn{3}{|c|}{ No. of metastatic nodal stations } \\
\hline$\leq 1$ & 18.7 & 0.06 \\
\hline$>1$ & 12.1 & \\
\hline \multicolumn{3}{|l|}{$\operatorname{LNR}(\%)$} \\
\hline$\leq 13$ & 19.9 & 0.01 \\
\hline$>13$ & 11.7 & \\
\hline \multicolumn{3}{|l|}{ Stage } \\
\hline $\mathrm{I} / \mathrm{II}$ & 37.9 & 0.005 \\
\hline III/IV & 12.7 & \\
\hline \multicolumn{3}{|l|}{ Histology } \\
\hline Epitheloid & 19.4 & 0.009 \\
\hline Non epitheloid & 10.4 & \\
\hline \multicolumn{3}{|l|}{ Adjuvant radiotherapy } \\
\hline Administrated & 20 & 0.01 \\
\hline Non administrated & 7.4 & \\
\hline
\end{tabular}

radiotherapy, median OS and 5-year survival were 38 months and $35.8 \%$, respectively. By contrast, for patients with $\mathrm{N}+$ status
Table III. Continued.

\begin{tabular}{llll}
\hline & \multicolumn{3}{c}{ Multivariate analysis } \\
\cline { 2 - 4 } Factors & HR & CI & P-value \\
\hline PS 1 & 0.72 & $(0.36-1.44)$ & 0.35 \\
pT>pT1 & 2.44 & $(0.81-7.30)$ & 0.11 \\
pN+ & 2.04 & $(0.89-4.69)$ & 0.09 \\
Metastatic LNR $>13 \%$ & 1.03 & $(0.45-2.39)$ & 0.94 \\
Stage III/IV & 1.28 & $(0.65-2.56)$ & 0.48 \\
R2 surgical resection & 2.15 & $(0.68-6.80)$ & 0.19 \\
Non epitheloid histology & 1.83 & $(0.69-4.90)$ & 0.23 \\
Adjuvant radiotherapy & 0.36 & $(0.19-0.68)$ & 0.001 \\
\hline PS, performance status; LNR, lymph node ratio. &
\end{tabular}

without adjuvant radiotherapy negative status, the median OS and 5 -year survival were 8 months and $0 \%$, respectively. The metastatic LNR was not statistically significant in the multivariate analysis.

Recurrence status information was available for 64 patients. Among them, 26 (40.6\%) died disease-free, 17 (26.5\%) had ipsilateral recurrence, $12(18.7 \%)$ contralateral recurrence, $7(10.9 \%)$ peritoneal dissemination and $1(1.5 \%)$ had pericardial recurrence. Local recurrence was not influenced by the $\mathrm{N}+$ status $(\mathrm{P}=0.09)$.

Postoperative course. Thirty- and 90-day mortality were 8 and $14.1 \%$, respectively. The median hospitalization length of stay was 18.5 days (range, 1-391). This was longer for right side disease ( 38 vs. 16 days, $\mathrm{P}=0.006$ ). Forty-one patients $(41.4 \%)$ had 1 or more postoperative morbidity (range, 1-4). Bronchopleural fistulas (BPF) occurred in 10 patients (10.1\%), all located in the right side $(\mathrm{P}=0.005)$. There was no evidence of a relation between occurrence of BPF and the total number of lymph nodes dissected in the mediastinum $(\mathrm{P}=0.71)$. Eleven patients $(11.1 \%)$ experienced contralateral lung infection. Cardiac complications were observed in 17 patients $(17.1 \%)$ and included atrial arrhythmia (8), pulmonary embolism (4), acute coronary syndrome (4) and pericarditis (1). Other rarer morbidities included hemorrhagic shock (4), recurrent nerve paralysis (4), septic shock (7) and ischemic colitis (1).

\section{Discussion}

Our study shows the typical demographics and epidemiology of any MPM cohort: mostly males, history of asbestos exposure, predominance of epithelial histology and advanced disease at presentation. With respect to $\mathrm{N}$ status, almost $1 / 3$ of our patients had $\mathrm{N}+$ disease. The latter was not related to the neoadjuvant treatment, as shown in literature (8-10) and despite the high proportion of this therapy in our study.

Lymphatic dissemination of pleural disease is less known than lymphatic dissemination of lung cancer. Nevertheless, the current staging system for MPM has the same nodal map 
as the lung cancer classification. Many have questioned the validity of this approach as the patterns of lymphatic pleural spread can affect the prognostic value of internal mammary or parietal nodal involvement, which could be different from the prognostic significance of axial mediastinal nodal metastasis (14). Focusing on the involvement of internal mammary nodes, our findings are similar to literature data with $6.5 \%$ (9) to $11.2 \%$ (8) of the patients presenting metastatic nodes at this level. On the other hand, only $5 \%$ had parietal nodes involved, in our study. Edwards et al (9) observed the same rate of involvement, but there is a lack of information on this issue in literature. One may expect a higher level of parietal node involvement in this primitive pleural disease but this may be underestimated by the absence of systematic nodal research in this large anatomic region. In addition, as the whole parietal pleura is closer to initial tumor site and probably invaded early, parietal nodes could be the first nodal relay and therefore be an N1 station and not an N3 one. Similarly N1 involvement in MPM may occur only after lung invasion, being a sign of a more aggressive disease than a 'simple' isolated N2 tumor (15).

Mediastinal nodal status was explored in the majority of our patients by the PET-CT scan couple. To better assess preoperative nodal involvement in 15 doubtful cases we used mediastinoscopy, which proved to be a reliable examination. All the 15 mediastinoscopy-negative patients were free of axial mediastinal lymph node involvement in the final histological findings. Only two of them had positive nodes in the parietal and internal mammary stations, which were not investigated by mediastinoscopy. Given the high specificity and the possible impact of N2 status on survival, mediastinoscopy should be proposed every time that result from thoracic CT and PET-CT scans are doubtful.

In this study, we aimed to clarify the potential mismatch between different $\mathrm{N}+$ status and to study the role of the metastatic LNR in MPM survival. This simple histological tool has shown great potential in other cancers $(16,18)$ but, to the best of our knowledge, LNR has not yet been evaluated on MPM. As others before (8) we confirm, in this study, that $\mathrm{N}+$ status was an important prognostic factor. Metastatic LNR showed a suggestive relation with OS at $13 \%$ cut-off level. The fact that this was related to survival while including the whole cohort ( $\mathrm{N} 0$ and $\mathrm{N}+$ patients) and not observed in the subgroup of $\mathrm{N}+$ patients, suggests that the survival of patients with few nodes involved, tends to be similar to N0 patient survival. This information seems more important than the location of the involved nodes. Although it failed to reach full conventional statistical significance in the multivariate analysis, we believe this ratio is more reliable than the number of involved lymph nodes $>3$ (9), involvement of superior mediastinal nodes (14) or the total number of dissected nodes, which display a wide range of variation determined by factors not necessarily related to disease or its prognosis, like BMI or neoadjuvant therapy.

Although neoadjuvant chemotherapy was more common in $\mathrm{R} 0$ surgical resection and that $\mathrm{R} 0$ resection influenced survival, neoadjuvant chemotherapy was not related to OS. By contrast, adjuvant radiotherapy was a very important prognostic factor. This relates to the necessity of reducing treatment morbidity thus permitting to deliver radiotherapy as quickly as possible.

Well known predictors of poor prognosis in MPM, described by Steele et al (24) such as poor PS and non- epitheloid histology were confirmed by our results, while the male gender was not significantly correlated to survival. Median OS and perioperative mortality were in the range of previously published systematic reviews of EPP for MPM (3).

Limitations of this study include its retrospective nature thus, introducing some degree of heterogeneity like the patients treated by tetramodal therapy, including HIT chemotherapy, instead of the standard trimodal therapy. Despite the fact that this cohort included 99 patients, the statistical power may be insufficient. This could be explained by the relatively small number of $\mathrm{N}+$ patients $(34.3 \%)$. Follow-up information was complete, but relevant information such as recurrence status was incomplete, not allowing a comprehensive analysis of the impact of nodal involvement. Plus, we studied patients from a 13-year period a factor possibly participating in the heterogeneity of this cohort. Similarly, there is a possible bias in the uniformity of pathological analysis which was done in four different centers.

In conclusion, except for $\mathrm{N}+$ status and metastatic LNR $>13 \%$, we did not observe any impact on OS on the number of involved nodes $>3$ nor on the involvement of superior mediastinal nodes. This follows the difficulty to unambiguously and reproducibly identify single prognostic factors also shown by previous studies where results were often inconsistent. We suggest that the metastatic LNR is likely to play an important role in the future assessment of the MPM patients, which should receive more attention and be further confirmed by larger prospective studies.

\section{Acknowledgements}

The authors are grateful to Claire Pinçon for her help with statistical analysis and to Dr Fabrice Kwiatkowski who kindly provided SEM software.

\section{References}

1. Robinson BW and Lake RA: Advances in malignant mesothelioma. N Engl J Med 353: 1591-1603, 2005.

2. Treasure T, Lang-Lazdunski L, Waller D, Bliss JM, Tan C, Entwisle J, et al: Extra-pleural pneumonectomy versus no extra-pleural pneumonectomy for patients with malignant pleural mesothelioma: clinical outcomes of the Mesothelioma and Radical Surgery (MARS) randomised feasibility study. Lancet Oncol 12: 763-772, 2011.

3. Cao CQ, Yan TD, Bannon PG and McCaughan BC: A systematic review of extrapleural pneumonectomy for malignant pleural mesothelioma. J Thorac Oncol 5: 1692-1703, 2010.

4. Van Schil P: Malignant pleural mesothelioma: staging systems. Lung Cancer 49: S45-S48, 2005.

5. Rusch VW: A proposed new international TNM staging system for malignant pleural mesothelioma. From the International Mesothelioma Interest Group. Chest 108: 1122-1128, 1995.

6. Boutin C, Dumortier P, Rey F, Viallat JR and De Vuyst P: Black spots concentrate oncogenic asbestos fibers in the parietal pleura. Thoracoscopic and mineralogic study. Am J Respir Crit Care Med 153: 444-449, 1996.

7. Okiemy G, Foucault C, Avisse C, Hidden G and Riquet M: Lymphatic drainage of the diaphragmatic pleura to the peritracheobronchial lymph nodes. Surg Radiol Anat 25: 32-35, 2003.

8. Flores RM, Routledge T, Seshan VE, Dycoco J, Zakowski M, Hirth Y and Rusch VW: The impact of lymph node station on survival in 348 patients with surgically resected malignant pleural mesothelioma: implications for revision of the American Joint Committee on Cancer staging system. J Thorac Cardiovasc Surg 136: 605-610, 2008.

9. Edwards JG, Stewart DJ, Martin-Ucar A, Muller S, Richards C and Waller DA: The pattern of lymph node involvement influences outcome after extrapleural pneumonectomy for malignant mesothelioma. J Thorac Cardiovasc Surg 131: 981-987, 2006. 
10. de Perrot M, Uy K, Anraku M, Tsao MS, Darling G, Waddell TK, et al: Impact of lymph node metastasis on outcome after extrapleural pneumonectomy for malignant pleural mesothelioma. J Thorac Cardiovasc Surg 133: 111-116, 2007.

11. Sugarbaker DJ, Flores RM, Jaklitsch MT, Richards WG Strauss GM, Corson JM, et al: Resection margins, extrapleural nodal status, and cell type determine postoperative long-term survival in trimodality therapy of malignant pleural mesothelioma: results in 183 patients. J Thorac Cardiovasc Surg 117: 54-65, 1999.

12. Chailleux E, Dabouis G, Pioche D, de Lajartre M, de Lajartre AY, Rembeaux Aand Germaud P: Prognostic factors in diffuse malignant pleural mesothelioma. A study of 167 patients. Chest 93: 159-162, 1988.

13. Aziz T, Jilaihawi A and Prakash D: The management of malignant pleural mesothelioma; single centre experience in 10 years. Eur J Cardiothorac Surg 22: 298-305, 2002.

14. Richards WG, Godleski JJ, Yeap BY, Corson JM, Chirieac LR, Zellos L, et al: Proposed adjustments to pathologic staging of epithelial malignant pleural mesothelioma based on analysis of 354 cases. Cancer 116: 1510-1517, 2010.

15. Abdel Rahman AR, Gaafar RM, Baki HA, El Hosieny HM, Aboulkasem F, Farahat EG, et al: Prevalence and pattern of lymph node metastasis in malignant pleural mesothelioma. Ann Thorac Surg 86: 391-395, 2008.

16. Nwogu CE, Groman A, Fahey D, Yendamuri S, Dexter E, Demmy TL, et al: Number of lymph nodes and metastatic lymph node ratio are associated with survival in lung cancer. Ann Thorac Surg 93: 1614-1620, 2012.

17. Wang CL, Li Y, Yue DS, Zhang LM, Zhang ZF and Sun BS: Value of the metastatic lymph node ratio for predicting the prognosis of non-small-cell lung cancer patients. World J Surg 36: 455-462, 2012
18. Mariette C, Piessen G, Briez N and Triboulet JP: The number of metastatic lymph nodes and the ratio between metastatic and examined lymph nodes are independent prognostic factors in esophageal cancer regardless of neoadjuvant chemoradiation or lymphadenectomy extent. Ann Surg 247: 365-371, 2008.

19. Wolf AS, Daniel J and Sugarbaker DJ: Surgical techniques for multimodality treatment of malignant pleural mesothelioma: extrapleural pneumonectomy and pleurectomy/decortication. Semin Thorac Cardiovasc Surg 21: 132-148, 2009.

20. Graham AN, Chan KJ, Pastorino U and Goldstraw P: Systematic nodal dissection in the intrathoracic staging of patients with non-small cell lung cancer. J Thorac Cardiovasc Surg 117: 246-251, 1999.

21. Naruke T, Suemasu K and Ishikawa S: Lymph node mapping and curability at various levels of metastasis in resected lung cancer J Thorac Cardiovasc Surg 76: 832-839, 1978.

22. Riquet M, Manac'h D, Dupont P, Dujon A, Hidden G and Debesse B: Anatomic basis of lymphatic spread of lung carcinoma to the mediastinum: anatomo-clinical correlations. Surg Radiol Anat 16: 229-238, 1994.

23. Mujoomdar AA and Sugarbaker DJ: Hyperthermic chemoperfusion for the treatment of malignant pleural mesothelioma. Semin Thorac Cardiovasc Surg 20: 298-304, 2008.

24. Steele JP, Klabatsa A, Fennell DA, Palläska A, Sheaff MT, Evans MT, et al: Prognostic factors in mesothelioma. Lung Cancer 49: S49-S52, 2005. 